\title{
A Study of the Mutual Diffusion Coefficient in a Binary Liquid Mixture, Using a Laser Beating Technique $\dagger$
}

\author{
F. M. EL-MEKAWEY
}

Physics Department, Faculty of Science, Tanta University, Tanta, A.R. Egypt

Line-widths of light scattered by nitrobenzene-heptane system are measured over a wide temperature range including the vicinity of the critical temperature of separation. The critical exponents are measured. Noncoincident value of the critical exponent for the states of the system above and below the critical temperature of separation are obtained.

\section{INTRODUCTION}

Light scattering with its recent development in laser optical mixing spectroscopy ${ }^{1,2}$ has become a very important tool for studying the thermodynamic properties in a binary liquid mixture.

Many equilibria and transport thermodynamic properties exhibit striking anomalies as the critical point is approached. Consequently, the determination of a symptotic laws governing the approach to a critical point is an important problem both from the experimental and theoretical point of view.

The purpose of the present work is to study the binary diffusion coefficient as a function of temperature.

\section{THEORETICAL}

The general theory of quasieleastic light scattering by fluid media has been well described in several excellent review articles. ${ }^{3,4}$ In particular,

† Work carried out in Molecular Physics Department, Moscow University, USSR. 
for a heterodyne scattering configuration when the field amplitude of the local oscillator beam is significantly greater than that of the light scattered by the medium, the spectrum of photocurrent developed by the detecting phototube is the convolution of the spectra of the local oscillator and scattered light beam.

For a binary liquid mixture it is well known that the frequency spectrum $S_{I}(\omega)$ of the intensity of the scattered light (and hence the frequency spectrum of the photocurrent) will have the form of a lorentzian shaped line whose half width $\Gamma$ depends on the binary diffusion coefficient $D$ and on the scattering angle as follows:

$$
S_{I}(\omega)=\Gamma(\theta) /\left|\omega^{2}+\Gamma^{2}(\theta)\right|
$$

where:

$$
\begin{gathered}
\omega=2 \Pi f \\
\Gamma(\theta)=D\left(\frac{4 \Pi n}{\lambda} \sin \frac{1}{2} \theta\right)^{2}
\end{gathered}
$$

and $\lambda$ is the wavelength of the incident light, $n$ is the index of refraction of the solution and $\theta$ is the scattering angle.

\section{EXPERIMENTAL}

A schematic diagram of our laser beat frequency spectrometer is shown in Figure 1. The experimental arrangement is essentially the same as that described in a previous work. ${ }^{5}$ Light from a coherent

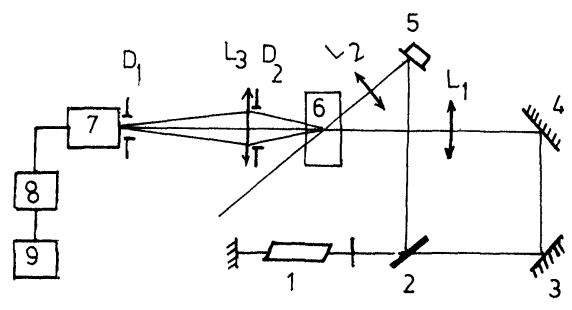

FIGURE 1 Experimental apparatus. 1. He-Ne laser, 2. Splitting plate, 3, 4, Mirrors, 5. Phase modulator, 6. Sample cell, 7. Photomultiplier tube, 8. Amplifier, 9. Spectrum analyzer. 
radiation $\mathrm{He}-\mathrm{Ne}$ laser (operating at $\lambda=6328 \AA$ with output power $30 \mathrm{MW}$ ) is split into two parts, the first one being focused in the sample, the other one is much lower in intensity, being used both as alignment beam and as a local oscillator in heterodyne light beating. The two parts are collected and focused onto the surface of the phototube. The photocurrent is amplified and fed to a spectrum analyzer, and the output of the averaged spectrum is recorded using an $X-Y$ recorder.

The pinholes $D_{1}$ and $D_{2}$ isolate a single coherence area in order to obtain a maximum signal/noise ratio. ${ }^{6}$ The sample was contained in a cylindrical glass cell housed in a temperature controlled copper jacket. During all measurements, the temperature was controlled to within $\pm 0.02 \mathrm{~K}$. Measurements have been performed on nitrobenzene-heptane mixture with concentration $X=53.28 \%$ wt. nitrobenzene (critical concentration $X_{c}=52.10 \%$ wt. nitrobenzene).

The measurements were carried out below and above the critical temperature of mixing. The maximum error in measurement of spectral half-width varied from 0.01 to 0.05 depending on the closeness to the critical mixing point.

\section{RESULTS AND DISCUSSION}

The temperature dependence of the half-width of the Rayleigh line (mutual diffusion coefficient) for nitrobenzene-heptane system at concentration $53.28 \%$ wt. nitrobenzene is graphically shown in Figure 2. Measurements were made along the critical isochore and the coexistance curve in the temperature range $5 \times 10^{-3} \leqslant \tau \leqslant 1$ where $\tau=T-T_{c} / T_{c}$ is the reduced temperature.

The data in all cases can be fit to a straight line plot of $\log \Gamma$ versus $\log (\tau)$. The analysis yields the following result:

$$
\Gamma=\Gamma_{0} \tau^{\nu}
$$

where $\nu$ is the critical exponent and $\Gamma_{0}$ is a numerical factor.

By combining Eqs. (1) and (2), we get:

$$
D=D_{0} \tau^{\nu}
$$

where $D$ is the mutual diffusion coefficient. 


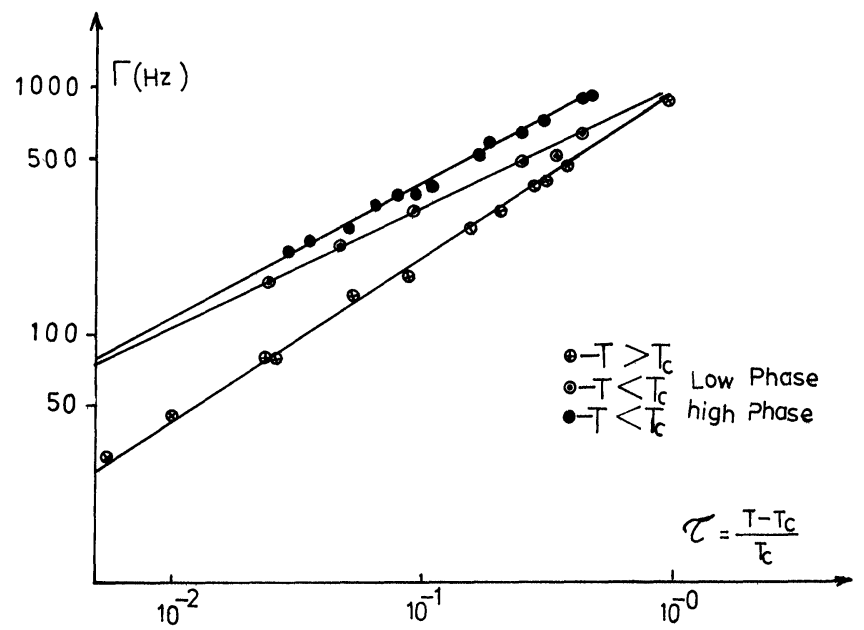

FIGURE 2 Temperature dependence of the Rayleigh half-width (mutual diffusion coefficient $)$ in nitrobenzene-heptane mixture. $(c=53.28 \%)$, the scattering angle $0=$ $13.25^{\circ}$.

The values of $D_{0}$ and $\nu$ obtained for homogeneous and heterogenous state of the system are given in the table. It should be note that the assymmetry behaviour $\left(\nu_{\text {high }}-\nu_{\text {low }}\right)$ in the present work is lower than the corresponding value in the previous work ${ }^{7}$ which has the value equal to 0.08 . This decrease in the asymmetry behaviour may be due to that the system was studied away from the critical concentration. The value of the critical exponent at $T>T_{c}$ is in a good agreement with those obtained by Ohbayashi, ${ }^{8}$ but we have noncoincident values of the critical exponent for the system in the two coexistance phase.

TABLE I

\begin{tabular}{lcc}
\hline Measuring range & $\nu$ & $D_{0} 10^{-6} \mathrm{~cm}^{2} / \mathrm{s}$ \\
\hline$T>T_{c}$ & $0.65 \pm 0.03$ & $4.98 \pm 0.2$ \\
$T<T_{c}$ & $0.49 \pm 0.05$ & $5.58 \pm 0.5$ \\
low phase & $0.53 \pm 0.03$ & $7.34 \pm 0.3$ \\
$\begin{array}{l}T<T_{c} \\
\text { high phase }\end{array}$ & & \\
\hline
\end{tabular}




\section{References}

1. H. Z. Cummins and H. L. Swinney, Progress in Optics, Vol. 8, ed. E. Wolf (Wiley, New York, 1970).

2. H. L. Swinney, Photon Correlation and Light Beating Spectroscopy, eds. H. Z. Cummins and E. R. Pike (Plenum Press, New York and London, 1974).

3. M. J. French, J. C. Angus and A. G. Walton, Science 163, 345 (1969).

4. B. Chu, Ann. Rev. Phys. Chem. 21, 145 (1970).

5. F. M. El-Mekawey, D. J. Sci. 6, (1982) to be published.

6. N. I. Chernova and S. V. Kazakov, Opt. and Spectro. (USSR), 49(2), 404-406 (1980).

7. N. I. Chernova, S. V. Kazakov and F. M. El-Mekawey, Vesnik MGO, Ser. Fis. Astro. (USSR) (1982) to be published.

8. K. Ohbayashi, S. Kagoshima and A. Ikushima, Phys. Lett. 44A, 129 (1973). 